\title{
GESTIÓN DE TENDENCIAS STEM EN EDUCACION SUPERIOR Y SU IMPACTO EN LA INDUSTRIA 4.0
}

\section{MANAGEMENT OF STEM TRENDS IN HIGHER EDUCATION AND ITS IMPACT ON INDUSTRY 4.0}

\author{
Rodolfo JIMÉNEZ LEÓN ${ }^{1}$ \\ Deneb Elí MAGAÑA MEDINA ${ }^{2}$ \\ Silvia Patricia AQUINO ZÚÑIGA ${ }^{3}$
}

\begin{tabular}{|lll|}
\hline Recibido & $:$ & 25.02 .2021 \\
Aceptado & $:$ & 22.06 .2021 \\
Publicado & $:$ & 02.08 .2021 \\
\hline
\end{tabular}

\begin{abstract}
RESUMEN: Se Identifican las tendencias en el ecosistema de formación en habilidades STEM (Ciencia, Tecnología, Ingeniería y Matemáticas) para su gestión en organizaciones educativas. Esta investigación documental refiere a un diseño deductivo, exploratorio y descriptivo a través de un análisis sistemático de la literatura. De acuerdo a cinco pasos: 1) formulación de preguntas de investigación; 2) ubicación de los estudios; 3) selección y evaluación de estudios; 4) análisis y síntesis; 5) propuesta de resultados sobre beneficios y dificultades, en un total de 15 artículos. Con la hermenéutica objetiva, se responde a las dimensiones: a) perspectivas de gestión y regulación legal del ámbito de la educación en las condiciones de 4.0 para acelerar su ritmo de crecimiento e implementar las nuevas oportunidades de su desarrollo; b) temas de modernización de la educación en las condiciones de Industria 4.0; c) formación de personal digital, innovaciones educativas y diversificación de servicios educativos; d) habilidades STEM para la industria 4.0, se muestran cinco tablas y cinco propuestas para su aplicación en las organizaciones educativas del nivel superior en América Latina. Para las conclusiones se establece un marco a corto plazo para la divulgación de la agenda ecosistema STEM y habilidades STEM en las prácticas online.
\end{abstract}

Palabras clave: Gestión, Industria de la información, Competencia profesional, Educación superior, Método de formación.

\begin{abstract}
Trends in the STEM (Science, Technology, Engineering and Mathematics) skills training ecosystem are identified for their management in educational organizations. This documentary research is based on a deductive, exploratory and descriptive design through a systematic analysis of the literature, according to five steps: 1) formulation of research questions; 2) location of studies; 3) selection and evaluation of studies; 4) analysis and synthesis; 5) proposal of results on benefits and difficulties, in a total of 15 articles. With objective hermeneutics, it responds to the dimensions: a) perspectives of management and legal regulation of the field of education in the conditions of 4.0 to accelerate its growth rate and implement the new opportunities for its development; b) issues of modernization of education in the conditions of Industry 4.0; c) training of digital personnel, educational innovations and diversification of educational services; d) STEM Skills for Industry 4.0. Five tables and five proposals are shown for their application in higher-level educational organizations in Latin America. For the conclusions, a short-term framework is established for the dissemination of the STEM ecosystem agenda and STEM skills in online practices.
\end{abstract}

Keywords: Management, Information industry, Occupational qualifications, Higher education, Training methods.

\footnotetext{
${ }^{1}$ Candidato a Doctor en Administración Educativa. Profesor Investigador. Universidad Juárez Autónoma de Tabasco (UJAT). Villahermosa, México. Email: rdojle@gmail.com. ORCID: https://orcid.org/0000-0003-2635-9479

${ }^{2}$ Doctora en Ciencias Administrativas. Profesora Investigadora. Universidad Juárez Autónoma de Tabasco (UJAT. Villahermosa, México. Email: deneb_72@yahoo.com. ORCID: https://orcid.org/0000-0002-8579-596X

${ }^{3}$ Doctora en Ciencias de la Educación. Profesora Investigadora. Universidad Juárez Autónoma de Tabasco (UJAT. Villahermosa, México. Email: saquinozuniga@gmail.com. ORCID: https://orcid.org/0000-0002-7223-8582
} 


\section{Journal of the Academy $|100|$}

\section{INTRODUCCIÓN}

El cambio global hacia un futuro del trabajo se define por una prospectiva en constante expansión de nuevas tecnologías, por nuevos sectores y mercados, por sistemas económicos globales. A causa de ello, las instituciones de educación superior requerirán de un liderazgo decidido para llegar a la formación del futuro de trabajo que satisfaga el potencial humano y cree una prosperidad ampliamente compartida (World Economic Forum, 2020).

La transición a la Industria 4.0 en las organizaciones educativas de nivel superior deberán proveer de transformación digital a través de la exploración de la conectividad, innovación de la experiencia, seguridad cibernética, inteligencia de datos en tiempo real, automatización y tecnología en nuevos campos. Estos avances, son el fundamento para el desarrollo de nuevas habilidades que permitan imaginar, entregar, y operar el futuro (Deloitte, 2019; Kraus, et al., 2019).

El término Industria 4.0 representa la cuarta revolución industrial (Gilchrist, 2016; Leminen et al., 2018; Rossit et al., 2018) y fue acuñado por primera vez en 2011, en Alemania (Frank et al., 2019; Tortorella et al., 2019). Dentro de esta revolución, existe una combinación esencial entre las tecnologías de Internet y las características de fabricación industrial optimizadas, por lo que el desarrollo de la competencia tecnológica en los docentes refiere a una necesidad urgente (World Economic Forum, 2020) en las organizaciones educativas, por lo que se debe diseñar, operar e implementar nuevos modelos de gestión del conocimiento.

En 2020, la globalización económica se encuentra estancada en América Latina, la cohesión social se verá erosionada por importantes disturbios y una polarización política, así mismo una recesión en desarrollo amenaza los medios de vida de quienes se encuentran en el extremo inferior del espectro de ingresos. A medida que una nueva recesión mundial provocada por la pandemia de salud COVID-19 afecta las economías y los mercados laborales, millones de los trabajadores ha experimentado cambios que han transformado profundamente su vida dentro y fuera del trabajo, así como su bienestar y su productividad (Banco Mundial, 2020, Fondo Monetario Internacional, 2020b; Secretaría de Hacienda y Crédito Público, 2020).

Dentro del contexto mexicano los programas prioritarios de asistencia social en educación superior por parte del Gobierno Federal se incluye dentro del presupuesto 2021, el 


\section{Journal of the Academy | 101 |}

otorgamiento deseable de becas a través de los programas: Becas Elisa Acuña con una apoyo anual correspondiente a 4,299.2 Millones de pesos corrientes en moneda nacional; para el ramo de formación y certificación para el trabajo, 3,775.2 y Universidades para el Bienestar Benito Juárez García, 1,019.4 aseguran la permanencia de los estudiantes en licenciatura e ingenierías en el territorio mexicano (Secretaría de Hacienda y Crédito Público, 2020).

El Consejo Nacional de Ciencia y Tecnología [CONACYT], sector del desarrollo de las innovaciones creadas por la actividad formal de Investigación y Desarrollo (I + D), a finales del mes de octubre del 2020 cancela fideicomisos, representando 500 millones de pesos anuales del presupuesto nacional. Estos cambios también se ven reflejados en el Sistema Nacional de Investigadores [SIN], restructurando políticas de ingreso, el cual busca la inclusión de mujeres y hombres que contribuyan a resolver los retos prioritarios del país a través de los Proyectos Nacionales de Investigación e Incidencia y los Programas Nacionales Estratégicos (ÁlvarezBuylla, 2020).

Ante los nuevos escenarios económicos, políticos y sociales, resultado de la rápida innovación tecnológica y su poder disruptivo, las organizaciones educativas de nivel superior, deberán replantearse las prácticas de enseñanza y aprendizaje, así como la adquisición de nuevos recursos tecnológicos; debido a los cambios fundamentales del nuevo siglo y la actualización de estudios de posgrado de acuerdo a las nuevas generaciones para la formación del futuro del empleo.

Con respecto a las nuevas generaciones, consideramos que esta adopción tecnológica, debe reflejarse en las habilidades: 1) Pensamiento analítico e innovación; 2) Aprendizaje activo y estrategias de aprendizaje; 3 ) Problema complejo: resolución; 4) Pensamiento crítico y análisis; 5) Creatividad, originalidad e iniciativa; 6) Liderazgo e influencia social; 7) Uso, seguimiento y control de la tecnología; 8) Diseño y programación de tecnología; 9) Resistencia, tolerancia al estrés y flexibilidad y 10) Razonamiento, resolución de problemas e ideación (World Economic Forum, 2020), las cuales deberán reflejarse en la educación en línea.

En relación con la educación en línea, esta ofrece numerosas ventajas y tiene el poder de superar las barreras tradicionales en la educación como el tiempo y el espacio a través de la práctica, el aprendizaje colaborativo como la vía para la búsqueda activa de información por 


\section{Journal of the Academy $|102|$}

parte de los estudiantes, la participación en la discusión, la formulación de preguntas y la discusión de respuestas, ideas y problemas desde diferentes perspectivas se ha adoptado como un nuevo paradigma de aprendizaje en contextos y dominios de aprendizaje dispares (Chow \& Shi, 2014).

Simultáneamente, con el apoyo, la orientación y el estímulo del docente, se busca la retroalimentación mutua, la crítica y la supervisión en el desempeño del estudiante; ante la evolución, de los programas de estudio de enfoques estandarizado a flexible, mixto o totalmente en línea, de independiente a colaborativo con el uso de diferentes plataformas y aplicaciones en la web, esta individualización en el entorno de aprendizaje está situada en la ontología (Reyes-Peña \& Tovar-Vidal, 2019) la cual se desarrolla a través de la web semántica, donde juega un papel de liderazgo en la construcción de un ecosistema de e-educación inteligente en las organizaciones educativas de nivel superior (Ouf, et al., 2017).

A su vez, la instrucción se centra más en el alumno, en lugar de centrarse en el docente, en consecuencia, también cambia el papel del profesor; en el nuevo proceso de aprendizaje, el docente a menudo se convierte en un facilitador en un entorno colaborativo al apoyar a los estudiantes en la creación de su conocimiento, por lo que el conocimiento se ve como un constructo social que se habilita a través de la interacción, evaluación y cooperación entre pares (Wieser \& Seeler, 2018), con el objetivo de proporcionar sugerencias prácticas e inspiración para implementar prospectivas del capital humano ante la transformación industrial 4.0, debiendo replantearse ¿cómo puede una institución educativa ante escenarios adversos configurar nuevos perfiles educativos con una planta docente antigua?

Simultáneamente la organización educativa en el nivel superior deberá aprovechar los registros de máquina de usuarios, los datos del sensor, imágenes fijas, video, audio, información biométrica, investigación del gobierno, participando de la realidad digital, la cadena de bloques, el negocio de la tecnología, la experiencia digital, el uso de la nube y analíticas para la competitividad de la organización educativa, estos datos permiten desarrollar el big data para la identificación de nuevos mercados y posicionamiento de las universidades.

Considerando la experiencia digital una línea de acción que permita a las organizaciones educativas, estudiantes, docentes, personal administrativo y comunidad universitaria un 


\section{Journal of the Academy $|103|$}

compromiso y para la gestión de los entornos digitales; el diseño centrado en lo humano y el compromiso del usuario se han vuelto piezas centrales de la estrategia de la educación online, el número de estudiantes inscritos en programas en línea ha crecido y a su vez también han cambiado los modelos pedagógicos y los sistemas de educación superior con el objetivo de utilizar el espacio virtual que ofrece Internet, transformándolo en un espacio de aprendizaje social (Harasim, 2000; Liang \& Chen, 2012; McKiernen \& Wilson, 2014), a través de la ciencia de las analísticas, centros educativos necesitan la capacidad para predecir y prescribir a través de la nube y la realidad digital.

De acuerdo con Ahmed et al. (2018), el mundo sigue inevitablemente las pautas de la Industria 4.0, los procesos convencionales serán reemplazados gradualmente por nuevos conceptos, tan solo en la gestión de documentos, con el uso de firmas digitales a través de los QR, el internet de las cosas [Internet of Things (IoT)], la realidad mixta [mixed reality (MR)], virtual [virtual reality (VR)], aumentada [augmented reality (AR)], tecnologías espaciales y experiencias inmersivas, actualmente están redefiniendo las prácticas administrativas, educativas y políticas de cómo los seres humanos interactúan en correlación con la evolución de la tecnología y los datos.

Con respecto a este panorama mundial, La National Science Foundation (2020), creó la noción del acrónimo STEM a fines de la década de 1990 con el objetivo de formar nuevas habilidades en las futuras generaciones de jóvenes en los Estados Unidos y responder favorablemente a la revolución tecnológica 4.0, estas políticas educativas generaron un fenómeno geopolítico las cuales fueron rápidamente adaptadas por el Reino Unido y los países asiáticos como Taiwán, Japón, China y Corea; desarrollando sistemas educativos de alto rendimiento, creando políticas nacionales a favor de la ciencia y la tecnología que permitieron el impulso de las universidades e industrias en esas regiones. De acuerdo con Blackley \& Howell (2015) el desarrollo de este movimiento generó un interés en los entornos terciarios para aumentar el número de matrículas en programas STEM; los gobiernos centraron la agenda política para el desarrollo de reformas educativas y científicas.

Alrededor de 31 años desde la creación del término STEM (National Science Foundation, 2020), se presta atención a la expansión y acceso al ecosistema educativo sin importar los orígenes, la raza, la etnia, el género, la religión y los niveles de ingresos, para que el capital 


\section{Journal of the Academy | 104 |}

humano de las nuevas generaciones pueda aprender las maravillas y posibilidades de STEM y así mantener el interés y pasión a lo largo de sus vidas. Este documento identifica el constructo ecosistema STEM como el entorno geográfico favorable en lo educativo, social, político y económico que impulsa a la innovación, la prosperidad y la competitividad global del capital humano a través de la formación en Ciencia, Tecnología, Ingeniería y Matemáticas, generando acceso, oportunidades, estímulos y herramientas de participación en la economía de la innovación y del progreso ante los cambios tecnológicos.

Ante esta oportunidad y las condiciones de falta de crecimiento del PIB promedio per cápita en América Latina (Fondo Monetario Internacional, 2020a) es necesario establecer y comparar los modelos educativos en donde la educación STEM ha generado buenos resultados en la formación de habilidades, desarrollo de estrategias empresariales y aplicación de leyes que favorezcan el entorno en ciencia y tecnología.

\section{DESARROLLO}

\section{MATERIALES Y MÉTODO}

El diseño de la investigación se centralizó en el paradigma cualitativo con un diseño deductivo, exploratorio y descriptivo utiliza a la técnica de revisión documental en revistas de alto impacto, para un análisis sistemático de la literatura situado en la educación STEM por sus siglas en inglés (Science, Technology, Engineering and Mathematics) en el nivel superior. Para ello se construyó a través del método deductivo, cinco pasos, para la construcción de la investigación documental: 1) formulación de preguntas de investigación; 2) ubicación de los estudios; 3) selección y evaluación de estudios; 4) análisis y síntesis; e informar y 5) utilizar los resultados de la investigación; los cuales se describen a continuación:

1) Para la formulación de las preguntas, se sigue el proceso de la hermenéutica objetiva (Flick, 2018), derivado del interés sobre la naturaleza del problema acción conforme a los objetivos antes descritos, se desarrolla el supuesto, el cuál especifica que las buenas prácticas internacionales permiten ser marcos de gestión educativa para proyectar nuevos métodos de formación por medio de la industria de la información en Latinoamérica, la especificación del contexto desarrolla la competencia profesional abordando espacios propicios para la gestión en tendencias STEM en las organizaciones 


\section{Journal of the Academy $|105|$}

educativas del nivel superior, para ello nos planteamos: ¿Cuáles son las perspectivas de gestión y regulación?, ¿cuáles son los temas de modernización de la educación?, ¿cómo se forma al personal digital?, ¿cuáles son las habilidades STEM? y ¿cómo se gestiona un modelo de formación en habilidades STEM?; para dar respuesta analítica a las dimensiones (Ver Tablas 1, 2, 3, 4 y 5) las cuales se describen en el presente documento.

2) La revisión sistemática de la literatura consideró el contexto de la Industria 4.0 de acuerdo a revista de alto impacto en el Reino Unido y países asiáticos, en estas se identificaron (15) beneficios y (13) dificultades, en un total de 15 artículos para el desarrollo del ecosistema STEM en América Latina.

3) Para la selección de los documentos se ingresó al portal Scimago Journal \& Country Rank, en donde se identificaron las revistas científicas posicionadas en el cuartil 1 en Educación de acuerdo a la categoría Ciencias Sociales y subárea en Administración Pública, procedente de los países en economías desarrollados por los ecosistemas STEM (Reino Unido y países asiáticos), a estas se les asigno un código axial de acuerdo a las respuestas formuladas para conocer su gestión a través de la investigación cualitativa, identificando: A1: Journal of Higher Education Policy and Management, A2: Studies in Higher Education, B1: Journal of Vocational Education \& Training, C1: Journal of Educational Administration, C2: Educational Research Review, D:4 Educational Studies in Mathematics y D2: British Educational Research Journal.

En el cuartil segundo se encontró a D6: Frontiers of Education in China y para el cuartil cuarto, se identificó a D4: Asia-Pacific Science Education. A su vez se consultó literatura gris, con los rangos de selección en editoriales de alto prestigio de acuerdo a los casos: D1: John Wiley \& Sons, Inc., D3: Routledge, D5: Springer Publishing. Así mismo para identificar el contexto en América Latina se abordó la revista D8: Revista de educación técnica integrada al Registro Nacional Científico, Tecnológico y de Innovación Tecnológica.

Cabe destacar que los criterios para la inclusión de los estudios en las revistas fue a través del uso de once palabras claves: 21th century skills, Education Reform, Epistemic Governance, Future Skills, Industry 4.0, Management, Performance 


\section{Journal of the Academy $|106|$}

Improvement, Productivity, STEM Education, STEM Skills y Technology Adoption (Habilidades del siglo XXI, Reforma Educativa, Gobernanza Epistémica, Habilidades futuras, Industria 4.0, Gestión, Mejora del rendimiento, Productividad, Educación STEM, Habilidades STEM y Adopción de Tecnología), las cuales permitieron seleccionar a los quince artículos de doscientos treinta durante el período del 2000 al 2020, considerando a la última ola tecnológica en las organizaciones para la transformación digital, propuestas por Hinchcliffe, (2015) denominada: Era de la Integración; así mismo se dio lectura a los 15 artículos en idioma inglés a través de sus resúmenes y contenidos para desarrollar las tablas de gestión documental.

4) A partir del análisis de los datos abiertos se identificaron las subcategorías que clasifican beneficios y dificultades en los ecosistemas STEM, de acuerdo a las dimensiones: A) Perspectivas de gestión y regulación legal del ámbito de la educación en las condiciones de 4.0 para acelerar su ritmo de crecimiento e implementar las nuevas oportunidades de su desarrollo. B) Temas de modernización de la educación en las condiciones de Industria 4.0 C) Formación de personal digital, innovaciones educativas y diversificación de servicios educativos y D) Formación de habilidades STEM (En español: Ciencia, Tecnología, Ingeniería y Matemáticas) en industria 4.0; la información obtenida permitió clasificarla a través de cinco tablas de datos cualitativos, las cuales incluyen las descripciones de las dimensiones, facilitando al lector la identificación de los códigos.

5) Con los resultados de la investigación documental se considera pertinente el contexto global para conocer la forma de gestionar un ecosistema STEM; para la internacionalización y conectividad de las palabras claves de la presente investigación se utilizan Tesauros de la United Nations Educational, Scientific and Cultural Organization (UNESCO, 2021). 


\section{Journal of the Academy | $107 \mid$}

\section{RESULTADOS}

Los recursos humanos en las organizaciones se consideran la principal fuente para la innovación, dada la aceleración continua del ciclo de desarrollo industrial, los cambios del mercado requieren de nuevos conocimientos y de nuevas inversiones tecnológicas; en países como Corea del Sur la industria manufacturera dejará de existir en los próximos diez años, este desempleo tecnológico debe reestructurar a la educación superior y ponderar los nuevos roles de gobierno, industria y creación del conocimientos para adaptarse; para Suecia las presiones hacia la creación de riqueza, la innovación y el crecimiento las organizaciones han desarrollado desafíos para la gobernanza epistémica en los entornos STEM; ante estos contextos surge la pregunta ¿cuáles son las perspectivas gestión y regulación que utilizan los países de Corea del Sur y Suecia en el ecosistema STEM?

Los procesos de cambio de políticas tienen lugar entre una amplia variedad de sectores y actores en una sociedad del conocimiento, incluidos el gobierno, la industria y las universidades. Esta vinculación genera sistemas de producción del conocimiento esperando que la cuarta revolución industrial traiga cambios disruptivos a la economía y la sociedad, los casos en Corea del Sur (Jung, 2019) y Suecia (Jacob \& Hellström, 2018), han intentado reformar sus sistemas de producción de conocimientos en los sectores público y privado y en las instituciones de educación superior coordinando importantes asuntos políticos en relación con la investigación y el desarrollo y selección de temas populares en la cuarta revolución industrial: inteligencia artificial, big data y biotecnología; estos temas se convierten en agendas de investigación nacional, la Tabla 1 identifica las dificultades que se presentan ante estos nuevos cambios. Se descubre que los instrumentos de financiación y el uso de agendas comunes, influyen en las reglas y procesos de la nueva gestión pública para las organizaciones de educación superior e investigación (Higher Education \& Research, HER por sus siglas en inglés) garantizando la validez y la calidad nacional. 
Journal of the Academy | 108 |

\section{Tabla 1}

Perspectivas de gestión y regulación legal del ámbito de la educación en las condiciones de 4.0 para acelerar su ritmo de crecimiento e implementar las nuevas oportunidades de su desarrollo

\begin{tabular}{|c|c|c|c|c|c|}
\hline Código & Autor & País & Beneficios & Dificultades & Enfocado a \\
\hline A1 & Jung (2019) & Corea del Sur & $\begin{array}{l}\text { Sistema de } \\
\text { producción de } \\
\text { conocimiento }\end{array}$ & $\begin{array}{l}\text { Desempleo } \\
\text { tecnológico, } \\
\text { debido a } \\
\text { reemplazo de } \\
\text { muchos puestos } \\
\text { de trabajo por } \\
\text { máquinas. }\end{array}$ & $\begin{array}{l}\text { Los académicos, } \\
\text { los responsables } \\
\text { políticos y los } \\
\text { líderes de la } \\
\text { educación } \\
\text { superior se guían } \\
\text { por una agenda } \\
\text { común. }\end{array}$ \\
\hline A2 & $\begin{array}{l}\text { Jacob y } \\
\text { Hellström } \\
(2018)\end{array}$ & Suecia & $\begin{array}{l}\text { Apoyar la } \\
\text { calidad en la } \\
\text { producción de } \\
\text { conocimiento } \\
\text { científico a } \\
\text { través de } \\
\text { agendas } \\
\text { comunes. }\end{array}$ & $\begin{array}{l}\text { Cambios en el } \\
\text { contexto en el que } \\
\text { se desarrolla la } \\
\text { política. } \\
\text { Presiones hacia la } \\
\text { creación de } \\
\text { riqueza, la } \\
\text { innovación y el } \\
\text { crecimiento. }\end{array}$ & $\begin{array}{l}\text { Gobernanza } \\
\text { epistémica como } \\
\text { algo que tiene que } \\
\text { ver con efectos } \\
\text { directos o } \\
\text { indirectos sobre la } \\
\text { producción, } \\
\text { selección y uso } \\
\text { del conocimiento } \\
\text { en la sociedad. }\end{array}$ \\
\hline
\end{tabular}

Fuente: Elaboración propia.

STEM e innovación son temas de modernización en las agendas internacionales para alcanzar los Objetivos del Desarrollo Sostenible (UNESCO, 2017) generar acceso igualitario, potenciar los derechos humanos y fortalecer las perspectivas científicas y de desarrollo para cuando la mano de obra no calificada sea más barata que las máquinas y están puedan sustituirla, se debe garantizar que niños, niñas, hombres y mujeres sean capaces de desarrollar habilidades STEM. Esto da como resultado la "paradoja de la productividad", por lo que es importante reconocer que la tecnología, la digitalización y la inteligencia artificial están entrelazadas con las relaciones sociales y, en consecuencia, son lugares de lucha de clases, brecha de género y acceso a comunidades. La forma en que esto se desarrolla es un resultado del equilibrio de poder, no solo dentro de la formación social sino también a nivel mundial, llegar a estos niveles de modernización con el caso de Reino Unido (Avis, 2018) se desarrollan a través de la concordia entre autoridades, y la disposición social. Por lo consiguiente, nuestra pregunta: 


\section{Journal of the Academy $|109|$}

¿Cuáles son los temas de modernización de la educación? Aborda la dirección en la inclusión social de individuos en el ecosistema STEM (Ver Tabla 2).

\section{Tabla 2}

Temas de modernización de la educación en las condiciones de Industria 4.0

\begin{tabular}{|c|c|c|c|c|c|}
\hline Código & Autor & País & Beneficios & Dificultades & Enfocado a \\
\hline B1 & Avis (2018) & $\begin{array}{l}\text { Huddersfield, } \\
\text { UK }\end{array}$ & $\begin{array}{l}\text { Tecnología e } \\
\text { inteligencia } \\
\text { artificial } \\
\text { entrelazadas a } \\
\text { través de las } \\
\text { relaciones } \\
\text { sociales. }\end{array}$ & $\begin{array}{l}\text { Enmarcarse } \\
\text { dentro de una } \\
\text { política más } \\
\text { amplia que } \\
\text { esté } \\
\text { comprometida } \\
\text { con el } \\
\text { desarrollo de } \\
\text { una sociedad } \\
\text { socialmente } \\
\text { justa. }\end{array}$ & $\begin{array}{l}\text { Los } \\
\text { académicos, } \\
\text { los } \\
\text { responsables } \\
\text { políticos y los } \\
\text { líderes de la } \\
\text { educación } \\
\text { superior. }\end{array}$ \\
\hline
\end{tabular}

Fuente: Elaboración propia.

La atención necesaria en el cambio de los modelos educativos y la aplicación de los financiamientos para generar infraestructura que favorezcan los ecosistemas de emprendimiento, países como Estados Unidos, Australia y los Países bajos, atienden en formación digital, la innovación educativa y la diversificación de entornos de aprendizaje colaborativo (Dijk, et al., 2020; Lioum, \& Daly, 2020; McClure, 2016; Wieser, \& Seeler, 2018). Ante la pregunta ¿cómo se forma al personal digital? Se identifican actividades académicas extracurriculares y tareas, desarrollo de confianza con la tecnología para el desarrollo de una identidad científica, regulado por prácticas en red y el aprendizaje reflexivo (Ver Tabla 3). 


\section{Journal of the Academy $|110|$}

\section{Tabla 3}

Formación de personal digital, innovaciones educativas y diversificación de servicios educativos

\begin{tabular}{|c|c|c|c|c|c|}
\hline Código & Autor & País & Beneficios & Dificultades & Enfocado a \\
\hline $\mathrm{C} 1$ & $\begin{array}{l}\text { Lioum \& } \\
\text { Daly, } \\
(2020)\end{array}$ & $\begin{array}{l}\text { California, } \\
\text { USA }\end{array}$ & $\begin{array}{l}\text { Práctica en red, } \\
\text { conexión y puente } \\
\text { entre organizaciones } \\
\text { dentro del ecosistema } \\
\text { STEM }\end{array}$ & $\begin{array}{l}\text { Modelos de } \\
\text { financiamiento } \\
\text { actuales en la } \\
\text { reforma } \\
\text { educativa } \\
\text { STEM }\end{array}$ & $\begin{array}{l}\text { Líderes a nivel } \\
\text { de sistemas, } \\
\text { Responsables } \\
\text { políticos y las } \\
\text { agencias } \\
\text { federales. }\end{array}$ \\
\hline
\end{tabular}

C2 Dijk, et Los Países Enseñar y apoyar el No presenta Docentes al.,(2020) Bajos aprendizaje reflexivo.

Diseño educativo.

Evaluación y retroalimentación.

Liderazgo y gestión educativa.

Investigación educativa.

Desarrollo profesional

\begin{tabular}{|c|c|c|c|c|}
\hline $\mathrm{C} 3$ & $\begin{array}{l}\text { Wieser, \& } \\
\text { Seeler } \\
\text { (2018). }\end{array}$ & Sidney & $\begin{array}{l}\text { aprendizaje } \\
\text { colaborativo en un } \\
\text { entorno de e-learning. }\end{array}$ & No presenta \\
\hline
\end{tabular}

\begin{tabular}{|c|c|c|c|c|}
\hline $\begin{array}{l}\text { McClure } \\
\text { (2016) }\end{array}$ & Ohio, EUA. & $\begin{array}{l}\text { Recaudando fondos, } \\
\text { estableciendo una } \\
\text { visión en torno al } \\
\text { espíritu empresarial y } \\
\text { cambiando las } \\
\text { políticas. }\end{array}$ & $\begin{array}{l}\text { Promover la } \\
\text { innovación y } \\
\text { el espíritu } \\
\text { empresarial } \\
\text { generan } \\
\text { conflicto con } \\
\text { los miembros } \\
\text { de la facultad. }\end{array}$ & $\begin{array}{l}\text { Capitalismo } \\
\text { académico } \\
\text { administrativo y } \\
\text { la capacidad } \\
\text { gerencial } \\
\text { extendida }\end{array}$ \\
\hline
\end{tabular}

Fuente: Elaboración propia. 


\section{Journal of the Academy $|111|$}

A medida que se aceleran los cambios en el trabajo, los empleadores son testigos de un cambio fundamental, el mercado laboral actual, el capital humano gira entre profesiones con conjuntos de habilidades significativamente diferentes y navegan por las transiciones laborales a mitad de carrera acompañadas de una actualización y mejora de habilidades sustanciales. Estas características son tan importantes para el éxito de las empresas como para la prosperidad ante esta afirmación indagamos ¿Cuáles son las habilidades STEM? (Ver tabla 4).

\section{Tabla 4}

Habilidades STEM (Ciencia, Tecnología, Ingeniería y Matemáticas) para la industria 4.0

\begin{tabular}{|c|c|c|c|c|c|c|}
\hline $\begin{array}{l}\text { Códig } \\
\text { o }\end{array}$ & Autor & País & Variable & Habilidades & Disciplina & Enfocado a \\
\hline D1 & $\begin{array}{l}\text { Lemaître, } \\
\text { (2018) }\end{array}$ & $\begin{array}{l}\text { Estados } \\
\text { Unidos }\end{array}$ & $\begin{array}{l}\text { Habilidades de } \\
\text { egreso }\end{array}$ & $\begin{array}{l}\text { Inteligencia social y } \\
\text { emocional, capacidad de } \\
\text { adaptación, sentido, } \\
\text { aprendizaje rápido, } \\
\text { pensamiento convergente } \\
\text { y divergente o } \\
\text { colaboración virtual, }\end{array}$ & Ingeniería & $\begin{array}{l}\text { Promover } \\
\text { Modelos } \\
\text { educativo } \\
\text { STEM }\end{array}$ \\
\hline D2 & $\begin{array}{l}\text { Ylonen, } \\
\text { (2012) }\end{array}$ & $\begin{array}{l}\text { Reino } \\
\text { Unido }\end{array}$ & $\begin{array}{l}\text { Habilidades } \\
\text { transferibles }\end{array}$ & $\begin{array}{l}\text { Comunicación, } \\
\text { presentación oral, } \\
\text { flexibilidad, confianza y } \\
\text { gestión del tiempo, } \\
\text { capacidad para trabajar } \\
\text { como miembro de un } \\
\text { equipo y bajo presión. }\end{array}$ & $\begin{array}{l}\text { Empleabili } \\
\text { dad }\end{array}$ & \\
\hline \multirow[t]{2}{*}{ D3 } & $\begin{array}{l}\text { Knight \& } \\
\text { Yorke } \\
(2003)\end{array}$ & $\begin{array}{l}\text { Reino } \\
\text { Unido }\end{array}$ & $\begin{array}{l}\text { Habilidades } \\
\text { básicas }\end{array}$ & $\begin{array}{l}\text { Aritmética, creatividad, } \\
\text { escucha y presentación } \\
\text { oral. }\end{array}$ & $\begin{array}{l}\text { Empleabili } \\
\text { dad }\end{array}$ & \\
\hline & & & $\begin{array}{l}\text { Habilidades de } \\
\text { proceso }\end{array}$ & $\begin{array}{l}\text { Planificación, influencia, } \\
\text { toma de decisiones y } \\
\text { trabajo en equipo. }\end{array}$ & & \\
\hline D4 & $\begin{array}{l}\text { Dogan \& } \\
\text { Tatsuoka, } \\
(2007)\end{array}$ & Turquía & $\begin{array}{l}\text { Habilidades } \\
\text { durante } \\
\text { evaluación }\end{array}$ & $\begin{array}{l}\text { Lidiaran con la } \\
\text { incertidumbre, derivaran } \\
\text { reglas y generalicen a } \\
\text { partir de casos, construyan } \\
\text { respuestas en lugar de } \\
\text { seleccionar una respuesta } \\
\text { de las alternativas dadas, } \\
\text { construyan respuestas en } \\
\text { lugar de seleccionar una }\end{array}$ & $\begin{array}{l}\text { Matemátic } \\
\text { as }\end{array}$ & \\
\hline
\end{tabular}


Journal of the Academy $|112|$

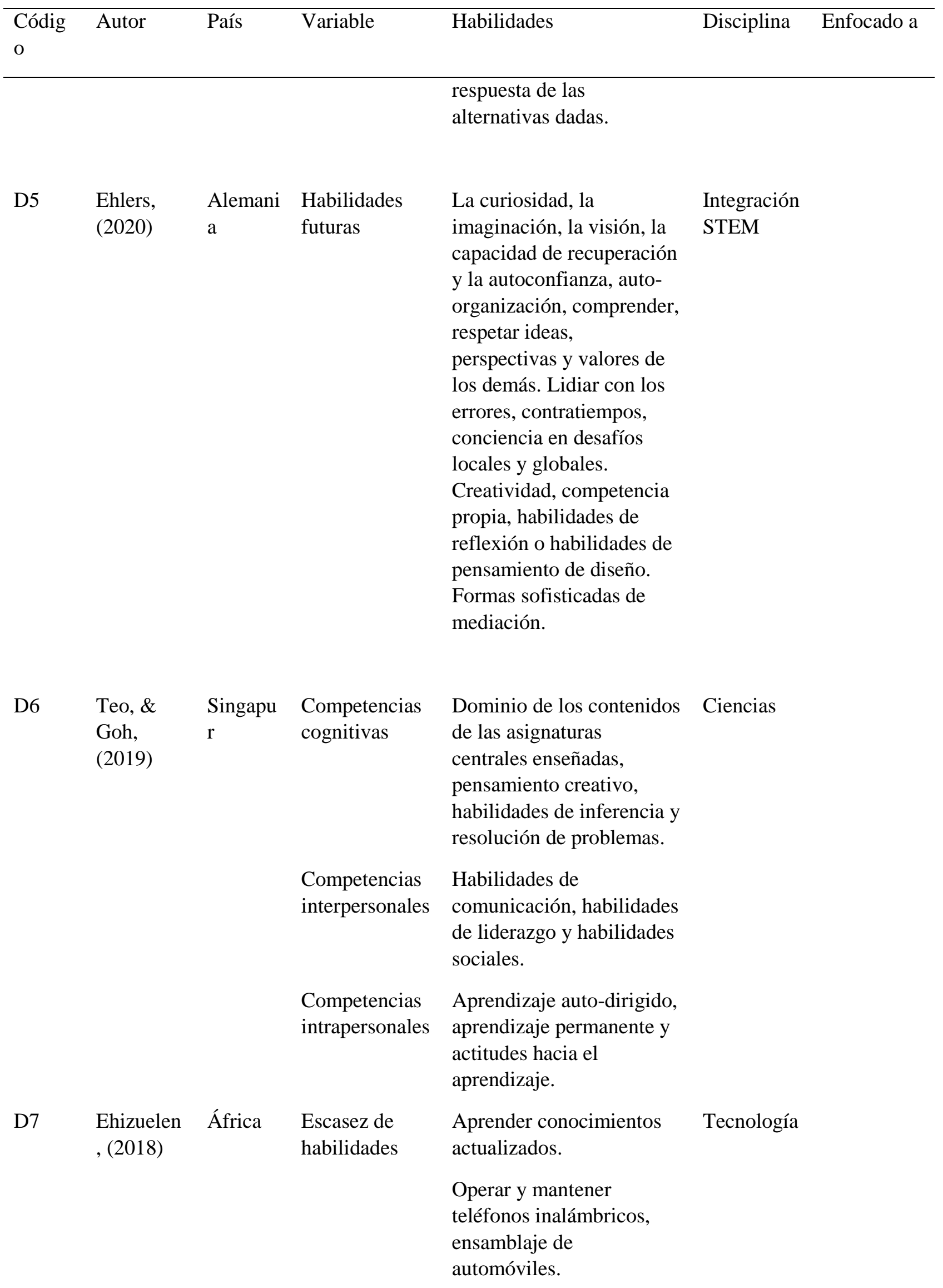


Journal of the Academy $|113|$

\begin{tabular}{|c|c|c|c|c|c|}
\hline $\begin{array}{l}\text { Códig } \\
\text { o }\end{array}$ & Autor & País & Variable & Habilidades & Disciplina \\
\hline D8 & $\begin{array}{l}\text { Jiménez- } \\
\text { León et. } \\
\text { al (2019) }\end{array}$ & México & $\begin{array}{l}\text { Habilidades } \\
\text { comunicativas } \\
\text { e } \\
\text { interpretativas }\end{array}$ & $\begin{array}{l}\text { Problematización y } \\
\text { cuestionamiento de } \\
\text { situaciones. }\end{array}$ & $\begin{array}{l}\text { Matemátic } \\
\text { as y } \\
\text { Tecnología }\end{array}$ \\
\hline
\end{tabular}

Fuente: Elaboración propia.

Las habilidades emergentes identificadas como de alta demanda dentro de las organizaciones de la industria 4.0 de acuerdo a World Economic Forum (2020) en México se distinguen: Resolución de problemas complejos; Aprendizaje activo y estrategias de aprendizaje; Pensamiento analítico e innovación; Pensamiento crítico y análisis; Diseño y programación de tecnología; Razonamiento, resolución de problemas e ideación; Creatividad, originalidad e iniciativa; Inteligencia emocional; Solución de problemas y experiencia del usuario; Orientación al servicio; Resiliencia, tolerancia al estrés y flexibilidad; Uso, seguimiento y control de la tecnología; Liderazgo e influencia social; Persuasión y negociación y Coordinación y gestión del tiempo presentes en el clima organizacional colaborativo, el cual deberá generarse estrategias de gestión del conocimiento a través de la aplicación de nuevos modelos, para dar respuesta a la pregunta: ¿Cómo se gestiona un modelo de formación en habilidades STEM?; se identifican acciones para su aplicación (Ver tabla 5).

\section{Tabla 5}

Análisis de estrategias para la gestión del conocimiento 
Journal of the Academy $|114|$

\begin{tabular}{|c|c|c|c|c|}
\hline \multicolumn{2}{|l|}{ Dimensiones } & \multirow{2}{*}{$\begin{array}{l}\text { Gestión del talento } \\
\text { humano }\end{array}$} & \multirow[t]{2}{*}{ Desempeño laboral } & \multirow[t]{2}{*}{ Estrategias } \\
\hline Clasificación & Descripción & & & \\
\hline \multirow[t]{2}{*}{ Habilidades } & $\begin{array}{l}\text { Diversificación } \\
\text { fuerza mental y } \\
\text { versatilidad. }\end{array}$ & $\begin{array}{l}\text { Creación de } \\
\text { Trabajos } \\
\text { completo, } \\
\text { coordinado y } \\
\text { flexibles con } \\
\text { claves de acceso } \\
\text { libre de los } \\
\text { integrantes del } \\
\text { grupo académico. }\end{array}$ & $\begin{array}{l}\text { Adaptación a } \\
\text { cambios y } \\
\text { situaciones extremas. }\end{array}$ & $\begin{array}{l}\text { Uso de la metodología } \\
\text { Blended Learning, } \\
\text { conocimientos } \\
\text { académicos de grupo y } \\
\text { uso de tecnología } \\
\text { responsable con docentes } \\
\text { y comunidad } \\
\text { universitaria. }\end{array}$ \\
\hline & $\begin{array}{l}\text { Más } \\
\text { intervención } \\
\text { creativa de las } \\
\text { autoridades. }\end{array}$ & $\begin{array}{l}\text { Comunidades } \\
\text { virtuales } \\
\text { organizadas a } \\
\text { través de agenda } \\
\text { electrónicas en } \\
\text { organización de } \\
\text { tiempos y } \\
\text { elección de } \\
\text { plataformas; con } \\
\text { las habilidades de } \\
\text { adopción, } \\
\text { aceptación de } \\
\text { nuevas ideas. }\end{array}$ & $\begin{array}{l}\text { Uso e incorporación } \\
\text { de nuevas } \\
\text { aplicaciones móviles. }\end{array}$ & $\begin{array}{l}\text { Integración } \\
\text { voluntariado } \\
\text { universitario a planta } \\
\text { administrativa, cuerpos } \\
\text { académicos y alumnado } \\
\text { para el fortalecimiento de } \\
\text { investigación en apoyo } \\
\text { social para la compasión } \\
\text { humanitaria. }\end{array}$ \\
\hline Motivación & Solidaridad & $\begin{array}{l}\text { Talleres y pláticas } \\
\text { virtuales. } \\
\text { Coaching virtual. }\end{array}$ & $\begin{array}{l}\text { Influencia dentro de } \\
\text { los grupos de trabajo. }\end{array}$ & $\begin{array}{l}\text { Creación de talleres y } \\
\text { pláticas virtuales con } \\
\text { temas de motivación, } \\
\text { coaching, entre otras. }\end{array}$ \\
\hline Actitudes & $\begin{array}{l}\text { Bienestar } \\
\text { colectivo. } \\
\text { Esfuerzo } \\
\text { colectivo. } \\
\text { Preservar sus } \\
\text { valores más } \\
\text { esenciales }\end{array}$ & $\begin{array}{l}\text { Campañas de } \\
\text { apoyo social. }\end{array}$ & Compasión humana. & $\begin{array}{l}\text { Mayor creatividad en el } \\
\text { uso de estrategias o } \\
\text { consignas para la } \\
\text { aplicación } \\
\text { conocimiento. } \\
\text { El docente presenta un } \\
\text { informe de sus avances, } \\
\text { metodologías, trabajo } \\
\text { colaborativo ante las } \\
\text { instancias } \\
\text { correspondientes. }\end{array}$ \\
\hline
\end{tabular}

Fuente: Elaboración propia de acuerdo con el uso de nuevas tecnologías para un clima organizacional colaborativo por Avendaño y Flores, (2016). 


\section{Journal of the Academy $|115|$}

\section{Propuestas para América Latina}

1. Fusionar la experiencia docente con personal antiguo y de nueva generación a través de los cuerpos académicos para contrarrestar el desplazamiento del capital humano ante la automatización.

2. Apostar a las nuevas tecnologías y la inteligencia artificial para contextos industriales, así como la producción de conocimiento alineada a un proceso sistemático que incluya identificar problemas regionales, creando nuevos diseños, priorizando y realizando inversiones circulares, que aseguren la protección del medio ambiente.

3. Favorecer redes entre gobierno, organización educativa y sociedad civil; a través de marcos jurídicos que faciliten su vinculación.

4. Promover el modelo STEM en toda América Latina, reestructurando desde la educación básica hasta la superior, con el fin de desarrollar nuevas habilidades que permitan a la nueva generación mayores proyecciones en la industria 4.0.

5. Crear marcos normativos donde se resguarde el derecho de las personas ante el uso de sus datos de registro online, con el uso de estos datos desarrollar marketing educativo a través de la identificación de intereses comunes que beneficien en la promoción de la institución, la región y al conocimiento.

\section{Discusiones}

La gestión de nuevos sistemas de producción de conocimiento y la evaluación de su calidad y pertinencia ante los nuevos paradigmas del nuevo siglo, en las organizaciones educativas de nivel superior deberán establecer estrategias a través de los cuerpos académicos para fusionar la experiencia del personal docente antiguo con los de nueva generación; optimizando recursos, respaldo y actualización el conocimiento.

De esta manera, ante las nuevas tecnologías y el desplazamiento del capital humano por la automatización, las autoridades educativas deberán atender los contextos territoriales de acuerdo con la investigación prioritaria nacional para obtener efectos directos o indirectos en los beneficios de la producción del conocimiento, esto refiere alinear las líneas de investigación con las agendas políticas y los intereses de las compañías industriales. 


\section{Journal of the Academy $|116|$}

Por lo tanto, la vinculación entre los gobiernos, empresas y universidades deberá ser una estrategia esencial para el desarrollo de nuevas tecnologías que beneficien a las zonas geográficas donde se encuentren, así mismo la creación de líneas de generación del conocimiento que respondan a las necesidades locales. Ante esta situación es necesario contar con tratados y marcos jurídicos que faciliten la vinculación para el desarrollo de una sociedad más justa; así mismo la revisión de reglamentos y contratos para las nuevas generaciones.

Con la incorporación del Modelo STEM en América Latina a mediano plazo, se busca formar a la nueva generación de la era de la síntesis que enfrentará no solo la automatización, si aún nuevos retos. Para corto plazo se busca promover las buenas prácticas docentes en entornos de e-learning fortaleciendo las habilidades en ciencia, tecnología, ingeniería y matemáticas, a través del diseño de cursos y talleres que permitan el fortalecimiento de las metodologías STEM para la solución de problemas de la comunidad virtual y la competencia internacional.

\section{CONCLUSIONES}

Ante los nuevos paradigmas de desarrollo económico en los países de América Latina, enseñar a las nuevas generaciones las habilidades académicas que desarrollen motivación y confianza en sus capacidades a través del modelo híbrido en disciplinas STEM (Fearon, et al. 2012), desafiando a los estereotipos de género sin importar los orígenes, raza, etnia, religión y nivel de ingresos, despertando la curiosidad e interés en proyectos, tareas innovadoras y tecnológicas, se busquen beneficiar a los países latinoamericanos, con el apoyo de una agenda en Educación Superior e Investigación para el desarrollo de políticas públicas con justicia social.

Conforme a lo anterior a corto plazo buscamos alentar a los administradores educativos, profesores investigadores, científicos, sociedad organizada a mejorar las oportunidades de progreso al avanzar en la educación STEM online (Allen \& Seaman, 2010; Harasim, 2000) en la comunidad virtual de acuerdo a las condiciones de confinamiento por la pandemia del Covid19; generar discusión a través de podcasts, talleres, foros de discusión y foros en línea (Fearon et al., 2012; Harasim, 2000; Liang \& Chen, 2012 y Moore, 1991) que permitan colocar en las agendas nacionales los temas del Ecosistema STEM y Habilidades STEM. 


\section{Journal of the Academy $|117|$}

Por lo tanto, conocer los ecosistemas STEM a través de la literatura citada en países como Alemania, Reino Unido, Singapur y Turquía (Dogan, \& Tatsuoka, 2007; Ehlers, 2020; Lemaitre, 2018; Teo \& Goh, 2019; Ylonen, 2012), nos permiten identificar las buenas prácticas para avanzar en los países latinoamericanos, con económicas en desarrollo como México (Jiménez-León et al., 2019), se recomienda indagar en los códigos abiertos identificados en las tablas antes descritas; para profundizar en estos modelos, y buscar su aplicación conforme a los contextos regionales, se propone redefinir los perfiles de egreso de las plantilla estudiantil en las universidades, reconociendo las necesidades del potencial humano (World Economic Forum, 2020) para un futuro presente; y así la programación de un nuevo modelo educativo STEM para el logro de los objetivos de Desarrollo Sostenible.

Las organizaciones educativas requieren de aplicar nuevas tendencias en recopilación de información, tratamiento y conversión de la información, así como la trasmisión eficiente de los contenidos virtuales. Para la investigación futura, se necesita de entrevistas con expertos para abordar sugerencias políticas, sociales, económicas y culturales, generando patrimonio a partir de sus activos intelectuales desarrollando una visión estratégica para potenciar el conocimiento y habilidades que generen ventajas competitivas, los estudios futuros deben incluir a la cuarta área industrial centrándose en cuestiones sociales, como la desigualdad social en los pueblos originarios y la protección del medio ambiente en el contexto latinoamericano, el cual está constituido por trabajadores del conocimiento competentes con capacidad de investigación que trasladan fronteras y sectores nacionales.

\section{REFERENCIAS BIBLIOGRÁFICAS}

Ahmed, M.B., Sanin, C. \& Szczerbicki, E. (2018). Experience-based decisional DNA (DDNA) to support product development, Cybernetics and Systems, 50(2), 399-411. https://doi.org/10.1080/01969722.2017.1418743

Álvarez-Buylla, M. (2020, 22 de octubre). Carta a las y los becarios e investigadores. (Reporte No.187). [Dirección General, C187/20]. Consejo Nacional de Ciencia y Tecnología. https://www.conacyt.gob.mx/index.php/comunicados/1327-com-187-2020

Allen, E., \& Seaman, J. (2010). Class Differences Online Education in the United States, 2010. Estados Unidos: Babson Survey Research Group. https://eric.ed.gov/?id=ED529952

Avendaño, P. V. y Flores, U. M. (2016). Modelos teóricos de gestión del conocimiento: descriptores, conceptualizaciones y enfoques. Entreciencias: Diálogos en la Sociedad del Conocimiento, 4(10), 201-227. https://www.redalyc.org/articulo.oa?id=4576/457646537004 
Avis, J. (2018). Socio-technical imaginary of the fourth industrial revolution and its implications for vocational education and training: a literature review. Journal of Vocational Education \& Training, 70(30), 337-363. https://doi.org/10.1080/13636820.2018.1498907

Banco Mundial. (2020, Junio 20). La COVID-19 (coronavirus) hunde a la economía mundial en la peor recesión desde la Segunda Guerra Mundial. https://www.bancomundial.org/es/news/press-release/2020/06/08/covid-19-to-plunge-globaleconomy-into-worst-recession-since-world-war-ii

Blackley, S., \& Howell, J. (2015). A STEM Narrative: 15 Years in the Making. Australian $\begin{array}{llll}\text { Journal of Teacher } & \text { Education, }\end{array}$ http://dx.doi.org/10.14221/ajte.2015v40n7.8

Chow, W. S., \& Shi, S. (2014). Investigating students' satisfaction and continuance intention toward E- learning: An extension of the expectation - Confirmation model. Procedia Social and Behavioral Sciences, 141(2014), 1145-1149. https://doi.org/10.1016/j.sbspro.2014.05.193

Deloitte (2019). Tendencias tecnológicas 2019, Más allá de la frontera digital. https://www2.deloitte.com/content/dam/Deloitte/co/Documents/technology/Tendencias_tecnolo gicas_2019\%20(Reporte\%20Completo).pdf

Dijk,E., Tartwijk,J., Schaaf,M. \& Kluijtmans, M. (2020). What makes an expert university teacher? A systematic review and synthesis of frameworks for teacher expertise in higher education. Educational Research Review, 31(100265), 1-84. https://doi.org/10.1016/j.edurev.2020.100365

Dogan, E., \& Tatsuoka, K. (2007). An international comparison using a diagnostic testing model: Turkish students' profile of mathematical skills on TIMSS-R. Educational Studies in Mathematics, 68(3), 263-272. https://doi.org/10.1007/s10649-007-9099-8

Ehizuelen, M. M. O. (2018). Education and Skills Development in China-Africa Cooperation. Frontiers of Education in China, 13(4), 553-600. https://doi.org/10.1007/s11516-018-0030$\underline{0}$

Ehlers, U. D. (2020). Future Skills. Springer Publishing. https://link.springer.com/book/10.1007/978-3-658-29297-3\#about

Fearon, C., Starr, S., \& McLaughlin, H. (2012). Blended learning in higher education (HE): conceptualising key strategic issues within a business school. Development and Learning in Organizations: An International Journal, 26(2), 19-22. $10.1108 / 14777281211201196$

Flick, U. (2018). Introducción a la investigación cualitativa (T. del Amo, Trad.). En E. Morata (Ed.), El procedimiento de la hermenéutica objetiva (pp. 222-223). (Obra original publicada en 2004). Fundación Paideia. 


\section{Journal of the Academy | $119 \mid$}

Fondo Monetario Internacional. (2020a, 13 de agosto). La falta de capital humano está frenando el crecimiento de América Latina [Comunicado de prensa]. https://www.imf.org/es/News/Articles/2020/08/12/na081320-lack-of-human-capital-is-holdingback-latin-americas-growth

Fondo Monetario Internacional. (2020b). Informes de perspectivas de la economía mundial. [Informe de políticas]. https://www.imf.org/es/Publications/WEO/Issues/2020/04/14/weoapril-2020

Frank, A.G., Dalenogare, L.S. \& Ayala, N.F. (2019), Industry 4.0 technologies: implementation patterns in manufacturing companies. International Journal of Production Economics, 210(9), 15-26. https://doi.org/10.1016/j.ijpe.2019.01.004

Gilchrist, A. (2016). Industry 4.0 the Industrial Internet of Things (1st ed.). Apress, Bangken. https://doi.org/10.1007/978-1-4842-2047-4

Harasim, L. (2000). Shift happens online education as a new paradigm in learning. The Internet and Higher Education, 3 (1), 41-61. https://doi.org/10.1016/S1096-7516(00)00032-4

Hinchcliffe, D. (2015, 10 de junio). How Should Organizations Actually Go About Digital Transformation? On Digital Strategy, Dion Hinchcliffe. https://dionhinchcliffe.com/2015/06/10/how-should-organizations-actually-go-about-digitaltransformation/

Jacob, M., \& Hellström, T. (2018). Epistemic governance and the conditions for knowledge production in HER institutions. Studies in Higher Education, 43(10), 1711-1717. https://doi.org/10.1080/03075079.2018.1520413

Jiménez-León, R., Magaña, D., Cisneros-Cohenour, E. y Aquino-Zuñiga, S. (2019). Apoyo educativo y de pares en las disciplinas de ciencia, tecnología, ingeniería y matemáticas. Revista de Educación Técnica, 3(9), 25-32. https://www.ecorfan.org/republicofperu/research_journals/Revista_de_Educacion_Tecnica/vol3 num9/Revista_de_Educaci\%C3\%B3n_T\%C3\%A9cnica_V3_N9_4.pdf

Jung, J. (2019). The fourth industrial revolution, knowledge production and higher education in South Korea, Journal of Higher Education Policy and Management, 12(6),134-156. https://doi.org/10.1080/1360080X.2019.1660047

Knight, P., \& Yorke, M. (2013). Learning, Curriculum and Employability in Higher Education (1ra ed.). Routledge. https://doi.org/10.4324/9780203465271

Kraus, S., Roig-Tierno, N., \& Bouncken, R. (2019). Digital innovation and venturing: An introduction into the digitalization of entrepreneurship. Review of Managerial Science, 13(3), 519-528. http://dx.doi.org/10.1007/s11846-019-00333-8

Lemaître, D. (2018). Training Engineers for Innovation. ISTE, Limited.

Leminen, S., Rajahonka, M., Westerlund, M. \& Wendelin, R. (2018). The future of the internet of things: toward heterarchical ecosystems and service business models. Journal of Business \& Industrial Marketing 33(6), 749-767. https://doi.org/10.1108/JBIM-10-2015$\underline{0206}$ 
Liang, R. \& Chen, D.-T. V. (2012). Online learning: Trends, potential and challenges. Creative Education, 3(8), 1332-1335. http://dx.doi.org/10.4236/ce.2012.38195

Lioum Y., \& Daly, A. (2020). Obstacles and opportunities for networked practice: a social network analysis of an inter-organizational STEM ecosystem. Journal of Educational Administration, 59(1), 94-115. https://doi.org/10.1108/JEA-02-2020-0041

McClure, K.R. (2016). Building the innovative and entrepreneurial university: An institutional case study of administrative academic capitalism. The Journal of Higher Education, 87(4), 516-543. https://doi.org/10.1080/00221546.2016.11777412

McKiernen, P., \& Wilson, D. (2014). Strategic choice: Taking business out of b-schools. En A. Pettigrew, E. Cornuel, \& U. Hommel (Eds.), The institutionial development of business schools (1st ed., pp. 248-269). Oxford University Press. https://strathprints.strath.ac.uk/56392/

Moore, M. G. (1991). Editorial: Distance education theory. American, Journal of Distance Education, 5(3), 1-6. https://doi.org/10.1080/08923649109526758

National Science Foundation. (2020). STEM Education for the future, a visioning report, may 2020. National Science Foundation a Subcommittee of the advisory committee of the education \& human resources directorate. https://www.nsf.gov/ehr/Materials/STEM\%20Education\%20for\%20the\%20Future\%20\%202020\%20Visioning\%20Report.pdf

Ouf, S., Abd-Ellatif, M., Salama, S. E., \& Helmy, Y. (2017). A proposed paradigm for smart learning environment based on semantic web. Computers in Human Behavior, 72(2017), 796-818. https://doi.org/10.1016/j.chb.2016.08.030

Reyes-Peña, C., \&Tovar-Vidal, M. (2019). Ontology: Components and Evaluation, a Review. $\begin{array}{llll}\text { Research in } \quad \text { Computing } & \text { Science, } 148 \quad \text { (3), }\end{array}$ https://www.rcs.cic.ipn.mx/2019_148_3/Ontologybased\%20Population\%20and\%20Enrichment\%200f\%20Researcher\%20Profiles.pdf

Rossit, D.A., Tohmé, F. \& Frutos, M. (2018), “Industry 4.0: smart scheduling”, International Journal of Production Research, $12 \quad$ (57), 3802-3813. https://doi.org/10.1080/00207543.2018.1504248

Secretaría de Hacienda y Crédito Público (2020). Documento relativo al cumplimiento de las disposiciones contenidos en el artículo 42, fracción I, de la Ley Federal de Presupuesto y Responsabilidad Hacendaria "Pre-Criterios2021". México: Gobierno de México. https://www.finanzaspublicas.hacienda.gob.mx/work/models/Finanzas_Publicas/docs/paquete_ economico/precgpe/precgpe_2021.pdf

Teo, T. \& Goh, W. (2019). Assessing lower track students' learning in science inference skills in Singapore. Asia-Pacific Science Education, 5(1), 1-19. https://doi.org/10.1186/s41029$\underline{019-0033-z}$ 


\section{Journal of the Academy $|121|$}

Tortorella, G.L., Giglio, R. y Van Dun, D.H. (2019). Industry 4.0 adoption as a moderator of the impact of lean production practices on operational performance improvement. International Journal of Operations \& Production Management, 39(6), 860-886. https://doi.org/10.1108/IJOPM-01-2019-0005

United Nations Educational, Scientific and Cultural Organization. (2021). Tesauro de la UNESCO (Versión 1) [Conjunto de datos]. Información del vocabulario [Gestión, Industria de la información, Competencia profesional, Educación superior, Método de formación]. https://vocabularies.unesco.org/browser/thesaurus/es/?clang=en

United Nations Educational, Scientific and Cultural Organization. (2017). Cracking the code: Girls and Women's education in science, technology, engineering and mathematics (STEM).

UNESCO. https://unesdoc.unesco.org/ark:/48223/pf0000253479?posInSet=20\&queryId=302e1749-d4144ce7-a4f0-b69fd3008e8f

Wieser, D., \& Seeler, J.-M. (2018). Online, Not Distance Education: The Merits of Collaborative Learning in Online Education. The Disruptive Power of Online Education, 125-146. https://doi.org/10.1108/978-1-78754-325-620181008

World Economic Forum (2020). The future of Jobs Report 2020. https://www.weforum.org/reports/the-future-of-jobs-report-2020

Ylonen, A. (2012). Student ambassador experience in higher education: skills and competencies for the future? British Educational Research Journal, 38(5), 801-811. https://doi.org/10.1080/01411926.2011.583636 